\title{
Field theory of self-avoiding walks in random media
}

\author{
A V Izyumov and K V Samokhin ${ }^{\dagger}$ \\ Cavendish Laboratory, Madingley Road, Cambridge, CB3 OHE, UK
}

(March 11, 2021)

\begin{abstract}
Based on the analogy with the quantum mechanics of a particle propagating in a complex potential, we develop a field-theoretical description of the statistical properties of a self-avoiding polymer chain in a random environment. We show that the account of the non-Hermiticity of the quantum Hamiltonian results in a qualitatively different structure of the effective action, compared to previous studies. Applying the renormalisation group analysis, we find a transition between the weak-disorder regime, where the quenched randomness is irrelevant, and the strong-disorder regime, where the polymer chain collapses. However, the fact that the renormalised interaction constants and the chiral symmetry breaking regularisation parameter flow towards strong coupling raises questions about the applicability of the perturbative analysis.
\end{abstract}




\section{INTRODUCTION}

The problem of a polymer in a random environment is among the most interesting in statistical physics. It has been known for a long time that the mean square end-to-end distance of a pure self-avoiding walk (SAW) of length $L$ obeys the scaling law $\left\langle r^{2}\right\rangle \sim L^{2 \nu}$, where $\nu \approx 0.59$ in three dimensions [1] (for a Gaussian random walk, one has the classical exact result $\nu=0.5 \mathrm{in}$ all dimensions). The question of how this scaling behaviour is affected by external impurities has attracted considerable research effort for more than a decade [5 [17]. In his pioneering work, Harris [6] argued that, treated perturbatively, quenched disorder is irrelevant, and, therefore, no modification of the critical exponent $\nu$ should be expected (see also Ref. [7]). This conclusion found support in the Monte-Carlo simulations on weakly diluted lattices [5]. The opposite case of strong disorder has also been studied, both numerically [8] and analytically [9, 10]. Edwards and Muthukumar [9] and Cates and Ball [10 predicted that a Gaussian chain placed in the field of impurities would collapse to a localised state, in which $\left\langle r^{2}\right\rangle \rightarrow$ const at $L \rightarrow \infty$. The localisation breaks down if one introduces a weak repulsive interaction between the monomers in the chain. In this case, it was found [11] that the polymer behaves as a free random walk with $\nu=0.5$. Later, a crossover between the regimes of weak $(\nu \approx 0.59)$ and strong $(\nu=0.5)$ disorder was predicted to occur at some critical concentration of impurities [12]. However, these results seem to contradict the conclusions of a number of other authors [14, 15], who argued that, at any concentration of impurities, the scaling of a long chain with excluded volume interactions is controlled by the critical exponent of a directed random walk, $\nu=2 / 3$. Overall, a comprehensive theory that would describe the effects of disorder on self-avoiding polymers is still missing.

Previous studies of the interplay of disorder and excluded volume interactions have been largely based on qualitative arguments, although a number of mathematical techniques (including the variational methods [11,12], replica field theories [7, 13, 14] and the real-space renormalisation group [16]) have also been used. A more general framework would therefore be beneficial to a better understanding of these effects. We believe that such a framework could be designed by employing a field-theoretical approach, which draws on the connection between the statistical mechanics of a polymer and the quantum mechanics of a particle in a complex random potential. Despite its apparent simplicity, the use of this method has been hampered by the fact that, as the Hamiltonian of the particle in a complex potential is non-Hermitian, it is impossible to represent the Green function in the form of a convergent functional integral (see below). A similar problem has been encountered, and successfully resolved, in the spectral theory of non-Hermitian operators. The latter has attracted great interest in recent years. A variety of applications have been identified including the study of anomalous diffusion in random media [18], scattering in open quantum systems [19], neural networks [20], and the statistical mechanics of flux lines in superconductors [21]. The problem of a self-avoiding walk without impurities, which can be mapped onto the quantum mechanics of a particle propagating in a random imaginary potential, has also been analysed in this context [22]. These studies have led to the development of a new technique, based on the representation of the spectral properties of non-Hermitian operators through an auxiliary Hermitian operator of twice the dimension [23], which serves as a good starting point for a field-theoretical approach. 
The main purpose of the present paper is to derive a consistent field-theoretical formulation of the problem of a self-avoiding polymer chain in a random white-noise potential, using the methods of non-Hermitian quantum mechanics, which is done in Section $\mathbb{1}$. The large-scale behaviour of this model is studied perturbatively in Section III by means of the momentum-shell renormalisation group (RG) in $D=4-\epsilon$ dimensions. Section [V] concludes with a discussion of the results obtained and of possible limitations on the validity of the perturbative approach.

\section{DERIVATION OF THE FIELD THEORY}

Let us consider a continuum self-avoiding chain of length $L$, with one end fixed at the origin. Then the probability to find the other end at a point $\mathbf{r}$ can be expressed as a path integral (Edwards model [24,25]):

$$
\begin{aligned}
P(\mathbf{r}, L)=\int_{\mathbf{x}(0)=\mathbf{0}}^{\mathbf{x}(L)=\mathbf{r}} \mathcal{D} \mathbf{x}(s) \exp \{ & -\frac{1}{2 a} \int_{0}^{L} d s\left(\frac{d \mathbf{x}(s)}{d s}\right)^{2}-\int_{0}^{L} d s V_{1}(\mathbf{x}(s)) \\
& \left.-\frac{\gamma_{2}}{2} \int_{0}^{L} d s_{1} \int_{0}^{L} d s_{2} \delta\left(\mathbf{x}\left(s_{1}\right)-\mathbf{x}\left(s_{2}\right)\right)\right\}
\end{aligned}
$$

The first term in the exponent corresponds to the entropic contribution (the length $a$, called the Kuhn length, is a microscopic parameter with the physical meaning of the monomer size, which provides a natural ultraviolet cutoff scale). The second term is the potential energy of the chain in an external potential, which is assumed to be a Gaussian distributed random function with the correlator $\overline{V_{1}(\mathbf{r}) V_{1}\left(\mathbf{r}^{\prime}\right)}=\gamma_{1} \delta\left(\mathbf{r}-\mathbf{r}^{\prime}\right)$. The last term takes into account the excluded volume effects $\left(\gamma_{2}>0\right)$, the limit $\gamma_{2} \rightarrow \infty$ describing the situation where the intersections of different fragments of the chain are penalized by an infinite energy barrier and thus completely forbidden. This interaction term can be decoupled by introducing an auxiliary white-noise potential. Using the identity

$$
\exp \left\{-\frac{\gamma_{2}}{2} \int_{0}^{L} d s_{1} \int_{0}^{L} d s_{2} \delta\left(\mathbf{x}\left(s_{1}\right)-\mathbf{x}\left(s_{2}\right)\right)\right\}=\left\langle\exp \left\{-i \int_{0}^{L} d s V_{2}(\mathbf{x}(s))\right\}\right\rangle
$$

where the angular brackets denote averaging over a Gaussian distributed random field $V_{2}(\mathbf{r})$ with correlator $\left\langle V_{2}(\mathbf{r}) V_{2}\left(\mathbf{r}^{\prime}\right)\right\rangle=\gamma_{2} \delta\left(\mathbf{r}-\mathbf{r}^{\prime}\right)$, we can represent Eq. (1) as an averaged Feynman propagator of a fictitious quantum particle moving in a complex random potential: $P(\mathbf{r}, L)=$ $\langle U(\mathbf{r}, L)\rangle$, where $\hat{U}(L)=\exp (-L \hat{H})$ with the Hamiltonian

$$
\hat{H}=-\nabla^{2}+V_{1}(\mathbf{r})+i V_{2}(\mathbf{r})
$$

$\left(V_{1,2}(\mathbf{r})\right.$ are independent Gaussian random fields). Note that $\hat{H}$ is non-Hermitian and may therefore have complex eigenvalues.

It is convenient to use the "energy representation", which is achieved by introducing the Green operator as a function of $z=x+i y$,

$$
\hat{g}(z) \equiv \frac{1}{z-\hat{H}}=\sum_{k}\left|R_{k}\right\rangle \frac{1}{z-z_{k}}\left\langle L_{k}\right|,
$$


where $\left|R_{k}\right\rangle$ and $\left\langle L_{k}\right|$ are the right and left eigenfunctions of $\hat{H}$, and $z_{k}$ denote the complex eigenvalues. Using the identity $\partial z^{-1} / \partial z^{*}=\pi \delta^{2}(z) \equiv \pi \delta(x) \delta(y)$, one can relate $\hat{U}(L)$ to $\hat{g}(z)$, and express the end-to-end probability distribution of a self-avoiding chain in a given distribution of impurities in the form

$$
P\left(\mathbf{r}, L \mid V_{1}\right)=\frac{1}{\pi} \int d^{2} z \exp (-z L) \frac{\partial}{\partial z^{*}}\langle g(\mathbf{r}, z)\rangle
$$

where the integration runs over the entire complex plane. It should be noted that the standard field-theoretical methods, based on the representation of an inverse matrix in the form of a Gaussian functional integral, cannot be directly used for the calculation of $\hat{g}(z)$, the reason being that, as $\hat{H}$ may have eigenvalues anywhere in the complex plane, it is impossible to guarantee the convergence of the functional integral. As pointed out in Ref. [26], a naive attempt to calculate the functional integral by the analytical continuation from the region where it converges to the whole complex plane fails. The problems are revealed by representing the density of complex eigenvalues through the identity

$$
\rho(z) \equiv \sum_{k} \delta^{2}\left(z-z_{k}\right)=\frac{1}{\pi} \frac{\partial}{\partial z^{*}} \operatorname{Sp} \hat{g}(z),
$$

wherein the Green function is shown to be non-analytic everywhere in which the density of states is non-vanishing.

To circumvent these difficulties, a representation has been introduced [23,27,28], in which the complex Green function $\hat{g}(z)$ is expressed through an auxiliary Hermitian operator, which in our case has the form

$$
\hat{G}^{-1}(z) \equiv\left(\begin{array}{cc}
0 & z-\hat{H} \\
z^{*}-\hat{H}^{\dagger} & 0
\end{array}\right)=\left(x+\nabla^{2}-V_{1}(\mathbf{r})\right) \sigma_{1}-\left(y-V_{2}(\mathbf{r})\right) \sigma_{2},
$$

where $\sigma_{i}$ are the Pauli matrices. A relationship between $\hat{g}$ and $\hat{G}$ is straightforward:

$$
\hat{g}(z)=\hat{G}^{21}(z)
$$

Using the replica trick, the matrix Green function $\hat{G}(z)$ can be written as a functional integral over $2 n$-component complex Bose fields (in the limit $n \rightarrow 0$ ):

$$
G^{i j}(\mathbf{r}, z)=-i \lim _{\eta \rightarrow+0} \int \mathcal{D}^{2} \boldsymbol{\varphi}_{a} \exp \left\{i \int d^{D} r \boldsymbol{\varphi}_{a}^{\dagger}\left(\hat{G}^{-1}(z)+i \eta \sigma_{0}\right) \boldsymbol{\varphi}_{a}\right\} \varphi_{1}^{i}(\mathbf{r}) \varphi_{1}^{j, *}(\mathbf{0})
$$

( $\sigma_{0}$ is the unit matrix, summation over repeated replica indices is assumed), where

$$
\boldsymbol{\varphi}_{a}(\mathbf{r})=\left(\begin{array}{l}
\varphi_{a}^{1}(\mathbf{r}) \\
\varphi_{a}^{2}(\mathbf{r})
\end{array}\right), \quad \mathcal{D}^{2} \boldsymbol{\varphi}_{a}=\prod_{a=1}^{n} \prod_{i=1,2} \frac{\mathcal{D}\left(\operatorname{Re} \varphi_{a}^{i}\right) \mathcal{D}\left(\operatorname{Im} \varphi_{a}^{i}\right)}{\pi}
$$

Due to the Hermiticity of $\hat{G}^{-1}(z)$ and the presence of the term with $\eta$ ("regulator"), the functional integral is well defined and convergent. Note that, although the matrix Green function (6) possesses a chiral symmetry, $\sigma_{3} \hat{G}^{-1}(z) \sigma_{3}=-\hat{G}^{-1}(z)$, this symmetry is broken in Eq. (8) if $\eta \neq 0$. 
Eqs. (7) and (8) allow one to average $\hat{g}(z)$ over $V_{2}$ and calculate the polymer partition function in a given configuration of the external disorder. In order to obtain physically observable quantities, one has to average the free energy $F(\mathbf{r}, L)=\ln P(\mathbf{r}, L)$ over the quenched random field $V_{1}$, which can be done using the replica trick once again:

$$
\begin{aligned}
\overline{F(\mathbf{r}, L)} & =\lim _{m \rightarrow 0} \frac{\overline{P^{m}(\mathbf{r}, L)}-1}{m} \\
& =\lim _{m \rightarrow 0} \frac{1}{m}\left\{\int \prod_{\alpha=1}^{m} \frac{d^{2} z_{\alpha}}{\pi} \exp \left(-L \sum_{\alpha=1}^{m} z_{\alpha}\right) \prod_{\alpha=1}^{m} \frac{\partial}{\partial z_{\alpha}^{*}} \overline{\prod_{\alpha=1}^{m}\left\langle g\left(\mathbf{r}, z_{\alpha}\right)\right\rangle}-1\right\} .
\end{aligned}
$$

It is thus necessary to introduce a second set of replica indices and to integrate over $2 \mathrm{~nm}$ component fields $\varphi_{a, \alpha}^{i}(\mathbf{r})(i=1,2 ; a=1, \ldots, n ; \alpha=1, \ldots, m)$. Averaging over $V_{1}$, we obtain from Eqs. (17) and (8):

$$
\overline{\prod_{\alpha}\left\langle g\left(\mathbf{r}, z_{\alpha}\right)\right\rangle}=\int \mathcal{D}^{2} \boldsymbol{\varphi}_{a, \alpha} e^{i S\left[\boldsymbol{\varphi}_{a, \alpha}^{\dagger}, \boldsymbol{\varphi}_{a, \alpha}\right]} \prod_{\alpha} \varphi_{1, \alpha}^{2}(\mathbf{r}) \varphi_{1, \alpha}^{1, *}(\mathbf{0}) \quad(n, m \rightarrow 0),
$$

where the effective action has the following form:

$$
\begin{aligned}
i S[\boldsymbol{\varphi}]=\int d^{D} r\{ & i\left(\boldsymbol{\varphi}_{a, \alpha}^{\dagger} \sigma_{1} \nabla^{2} \boldsymbol{\varphi}_{a, \alpha}\right)+i x_{\alpha}\left(\boldsymbol{\varphi}_{a, \alpha}^{\dagger} \sigma_{1} \boldsymbol{\varphi}_{a, \alpha}\right)-i y_{\alpha}\left(\boldsymbol{\varphi}_{a, \alpha}^{\dagger} \sigma_{2} \boldsymbol{\varphi}_{a, \alpha}\right)-\eta\left(\boldsymbol{\varphi}_{a, \alpha}^{\dagger} \sigma_{0} \boldsymbol{\varphi}_{a, \alpha}\right) \\
& -\frac{\gamma_{1}}{2}\left(\boldsymbol{\varphi}_{a, \alpha}^{\dagger} \sigma_{1} \boldsymbol{\varphi}_{a, \alpha}\right)\left(\boldsymbol{\varphi}_{b, \beta}^{\dagger} \sigma_{1} \boldsymbol{\varphi}_{b, \beta}\right)-\frac{\gamma_{2}}{2}\left(\boldsymbol{\varphi}_{a, \alpha}^{\dagger} \sigma_{2} \boldsymbol{\varphi}_{a, \alpha}\right)\left(\boldsymbol{\varphi}_{b, \alpha}^{\dagger} \sigma_{2} \boldsymbol{\varphi}_{b, \alpha}\right) \\
& \left.-\frac{\gamma_{1}^{\prime}}{2}\left(\boldsymbol{\varphi}_{a, \alpha}^{\dagger} \sigma_{1} \boldsymbol{\varphi}_{a, \alpha}\right)\left(\boldsymbol{\varphi}_{b, \alpha}^{\dagger} \sigma_{1} \boldsymbol{\varphi}_{b, \alpha}\right)\right\} .
\end{aligned}
$$

This action is different from the replica $n$-vector model, used previously in the RG analysis of the problem of a SAW in a random medium [7, 13, 14, because of the double dimensionality of the fields involved and a larger number of the coupling constants (three instead of two). The additional term proportional to $\gamma_{1}^{\prime}$ cannot be obtained in a formal derivation of Eq. (11), but should be added to the long-wavelength effective action for consistency, as we shall see below. Note also that we have kept the term with $\eta$ in Eq. (11) (the importance of this term will become clear shortly). The asymmetry between the ways the Latin and Greek replica indices appear in Eq. (11) reflects the differences in the nature of the random potentials $V_{1,2}$ : $V_{1}$ describes the external quenched disorder, while $V_{2}$ is a fictitious annealed random field.

\section{RENORMALISATION GROUP ANALYSIS}

The long-distance properties of the field theory (11) can be investigated using the momentum shell RG approach and the $\epsilon$-expansion near the upper critical dimension $D_{c}=4$. Using the standard procedure, consisting of the separation of "slow" and "fast" degrees of freedom followed by a rescaling of lengths and fields [29], we obtain the flow equations in one-loop order: 


$$
\begin{aligned}
& \frac{d \ln x_{\alpha}}{d \xi}=2+\frac{1}{8 \pi^{2}}\left(\gamma_{1}-\gamma_{2}+\gamma_{1}^{\prime}\right) \\
& \frac{d \ln y_{\alpha}}{d \xi}=2+\frac{1}{8 \pi^{2}}\left(\gamma_{1}-\gamma_{2}+\gamma_{1}^{\prime}\right) \\
& \frac{d \ln \eta}{d \xi}=2+\frac{1}{8 \pi^{2}}\left(\gamma_{1}+\gamma_{2}+\gamma_{1}^{\prime}\right) \\
& \frac{d \gamma_{1}}{d \xi}=\epsilon \gamma_{1}+\frac{1}{4 \pi^{2}}\left(2 \gamma_{1}^{2}-\gamma_{1} \gamma_{2}+\gamma_{1} \gamma_{1}^{\prime}\right) \\
& \frac{d \gamma_{2}}{d \xi}=\epsilon \gamma_{2}+\frac{1}{4 \pi^{2}}\left(3 \gamma_{1} \gamma_{2}-\gamma_{2}^{2}+3 \gamma_{2} \gamma_{1}^{\prime}\right), \\
& \frac{d \gamma_{1}^{\prime}}{d \xi}=\epsilon \gamma_{1}^{\prime}+\frac{1}{4 \pi^{2}}\left(3 \gamma_{1} \gamma_{1}^{\prime}+\gamma_{2}^{2}-\gamma_{2} \gamma_{1}^{\prime}+2 \gamma_{1}^{\prime 2}\right),
\end{aligned}
$$

where $\xi=\ln (L / a)$ is the logarithmic $R G$ parameter. The real and imaginary components of the complex "energy" $z=x+i y$ are renormalised in the same way. It also follows from Eqs. (12) that, even though initially $\gamma_{1}^{\prime}=0$, it acquires a non-zero value under renormalisation. Introducing a different set of independent variables,

$$
u=\gamma_{1}-\gamma_{2}+\gamma_{1}^{\prime}, \quad v=\gamma_{2}, \quad w=\gamma_{1}^{\prime},
$$

we rewrite Eqs. (12) as

$$
\begin{aligned}
& \frac{d \ln x}{d \xi}=2+\frac{1}{8 \pi^{2}} u, \quad \frac{d \ln y}{d \xi}=2+\frac{1}{8 \pi^{2}} u \\
& \frac{d \ln \eta}{d \xi}=2+\frac{1}{8 \pi^{2}}(u+2 v), \\
& \frac{d u}{d \xi}=\epsilon u+\frac{1}{2 \pi^{2}} u^{2}, \\
& \frac{d v}{d \xi}=\epsilon v+\frac{1}{4 \pi^{2}}\left(3 u v+2 v^{2}\right), \\
& \frac{d w}{d \xi}=\epsilon w+\frac{1}{4 \pi^{2}}\left(3 u w+v^{2}+2 v w-w^{2}\right) .
\end{aligned}
$$

Note that the flow equations for $x, y$ and $u$ are decoupled from those for $v$ and $w$.

Let us neglect the renormalisation of $\eta$, i.e. put $\eta_{0}=0$ (the consequences of allowing for $\eta_{0} \neq 0$ will be discussed below). As seen from Eq. (9), the main contribution to the average free energy at $L \rightarrow \infty$ comes from $x, y \sim 1 / L$, which provide the initial conditions for the equations (13). The scale $R_{c}$, at which the renormalised values of $x$ and $y$ become of the order of unity, represents the correlation length of our field theory and should be identified with the average size of a polymer of length $L$. Integrating Eqs. (13) with respect to $\xi$ from $\xi=0$ to $\xi_{c}=\ln \left(R_{c} / a\right)$, we obtain the equation which relates the polymer size to $L$ :

$$
\ln \frac{L}{a}=\int_{0}^{\xi_{c}} d \xi\left(2+\frac{u(\xi)}{8 \pi^{2}}\right) .
$$

The behaviour of $u(\xi)$ essentially depends on the bare values of the coupling constants $\gamma_{1,2}$ (the bare value of $\gamma_{1}^{\prime}$ is zero). One should distinguish between the two possibilities: In 
the weak disorder regime $\left(\gamma_{1}<\gamma_{2}\right.$, i.e. $\left.u_{0}<0\right), u(\xi)$ flows towards the stable fixed point $u^{*}=-2 \pi^{2} \epsilon$. After substitution in Eq. (18), we obtain $R_{c}(L) \sim L^{\nu}$ with $\nu \approx 1 / 2+\epsilon / 16$. This exponent coincides with the result for a pure SAW [1] which means that weak disorder is irrelevant for the large-scale properties of polymers. This result is in agreement with Refs. 6, 77.

In the strong disorder regime $\left(\gamma_{1}>\gamma_{2}\right.$, i.e. $\left.u_{0}>0\right)$, the solution of Eq. (15)

$$
u(\xi)=u_{0} \frac{e^{\epsilon \xi}}{1-\frac{u_{0}}{2 \pi^{2} \epsilon}\left(e^{\epsilon \xi}-1\right)}
$$

has a pole at $\xi=\xi^{*}$, where

$$
\xi^{*}=\frac{1}{\epsilon} \ln \left(1+\frac{2 \pi^{2} \epsilon}{u_{0}}\right) .
$$

Substituting $u(\xi) \sim\left(\xi^{*}-\xi\right)^{-1}$ in Eq. (18), we find that, at $L \rightarrow \infty, R_{c}(L) \rightarrow a e^{\xi^{*}}$. Thus, the polymer size tends to some constant independent of its length. Although this conclusion coincides with the results of Refs. 99,16.17], the applicability of our one-loop RG equations in this strong-coupling regime is limited.

\section{CONCLUSIONS AND DISCUSSION}

Our results show that a proper account of the non-Hermiticity leads to significant changes in the structure of the effective field theory for self-avoiding walks in random media. The application of the one-loop renormalisation group analysis to the action with the doubled number of the degrees of freedom allowed us to predict the existence of a phase transition between the weak disorder regime (in which the scaling behaviour of the polymer is not affected by the external randomness), and the strong disorder regime (in which the polymer collapses into a localised state). This conclusion seems to be in agreement with one of the scenarios considered previously, notably in Refs. [7, 16].

However, our RG calculations also indicate that there might be some hidden flaws within the perturbative approach, one of which is revealed by taking into consideration the infinitesimal regulator $\eta$, which explicitly breaks the chiral symmetry of Eq. (6). It follows from Eq. (16) that, in the weak disorder case, $v(\xi)$ has a pole at $\xi=\xi_{v}^{*}$, where

$$
\xi_{v}^{*}=\frac{1}{\epsilon} \ln \left(1+2 \pi^{2} \epsilon \frac{4 v_{0}-\left|u_{0}\right|}{\left(2 v_{0}-\left|u_{0}\right|\right)^{2}}\right) .
$$

After substitution in Eq. (14) and integration over $\xi$, we find that $\eta(\xi)$ grows under renormalisation, $\eta(\xi) \simeq \eta_{0}\left(1-\xi / \xi_{v}^{*}\right)^{-\epsilon / 2}$, and diverges at some scale $R_{c, \eta} \sim a e^{\xi_{v}^{*}}$. Although $\eta$ does not appear explicitly in the flow equations for $x, y$ and $u$, its singular $\mathrm{RG}$ behaviour may be an evidence of the spontaneous breaking of the chiral symmetry [30. In the fieldtheoretical language, it would manifest itself as the appearance of the anomalous pairings such as $\left\langle\varphi^{1, *} \varphi^{1}\right\rangle$ and $\left\langle\varphi^{2, *} \varphi^{2}\right\rangle$, which could change the long-distance behaviour of our system at scales larger than $R_{c, \eta}$. It might be the case that a seemingly trivial technical trick 
of doubling the degrees of freedom actually reveals deep physics related to the presence of the additional continuous symmetries in the system, whose effects would otherwise be completely lost.

Furthermore, despite the presence of a stable fixed point in the RG equation (15) for $u$ in the weak disorder regime, the coupling constants $\gamma_{1}, \gamma_{2}$ and $\gamma_{1}^{\prime}$ are all renormalised to infinity at large scales (see Eqs. (16) and (17)). The existence of the underlying RG flows towards a strong coupling regime can cast doubts in the validity of the scaling description itself (the authors of Refs. [14,15] suggested that the behaviour of the system could instead be determined by a strong-coupling fixed point, which is inaccessible by means of the perturbation theory). It is interesting that an analogous situation takes place even in the absence of an external disorder (i.e. at $\gamma_{1}=0$ ), in which case $\gamma_{2}$ and $\gamma_{1}^{\prime}$ both go to infinity, but $\gamma_{2}-\gamma_{1}^{\prime} \rightarrow 2 \pi^{2} \epsilon$. One of the possible explanations is that an important role could be played by the so-called "tail states" in the spectrum of the Hamiltonian (2), which would significantly change the observable large-scale behaviour of the system. The properties and physical manifestations of the "tail states" of Hermitian operators have been extensively studied since the pioneering works of I. M. Lifshitz [31]. A generalisation of this theory to the case of non-Hermitian random operators presents an interesting theoretical challenge with numerous potential applications.

Finally, we would like to emphasize that, since at every step of the calculations we deal with convergent functional integrals, our field-theoretical model of SAWs in random media is not only capable of reproducing some of the previous results on the perturbative level, but also seems to suit well for the study of non-perturbative effects, such as the chiral symmetry breaking and/or the contribution of the spectral "tails". We leave the investigation of such effects for future work.

\section{ACKNOWLEDGEMENTS}

The authors would like to thank B. Simons for numerous stimulating discussions, I. Lerner for insightful comments and communicating to us his unpublished results, and A. Moroz for interest to this work. K. V. S. is also pleased to thank A. Comtet and S. Nechaev

for interesting discussions and suggestions. The financial support by the Engineering and Physical Sciences Research Council, UK (K. V. S.) and Trinity College, Cambridge, UK (A. V. I.) is gratefully acknowledged. 


\section{REFERENCES}

$\dagger$ Permanent address: L. D. Landau Institute for Theoretical Physics, Kosygina Str. 2, 117940 Moscow, Russia.

[1] De Gennes P G 1972 Phys. Lett. 38A 339

[2] Emery V J 1975 Phys. Rev. B 11239

[3] Zinn-Justin J 1996 Quantum Field Theory and Critical Phenomena (Oxford: Clarendon Press)

[4] Kremer K, Baumgärtner A and Binder K 1981 Z. Phys. B 40331

[5] Kremer K 1981 Z. Phys. B 45149

[6] Harris A B 1983 Z. Phys. B 49347

[7] Kim Y 1983 J. Phys. C: Solid State Phys. 161345

[8] Baumgärtner A and Muthukumar M 1987 J. Chem. Phys. 873082

[9] Edwards S F and Muthukumar M J 1988 J. Chem. Phys. 892435

[10] Cates M E and Ball R C 1988 J. Physique 492009

[11] Edwards S F and Chen Y 1988 J. Phys. A: Math. Gen. 212963

[12] Thirumalai D 1988 Phys. Rev. A 37269

[13] Machta J and Kirkpatrick T R 1990 Phys. Rev. A 415345

[14] Obukhov S P 1990 Phys. Rev. A 422015

[15] Le Doussal P and Machta J 1991 J. Stat. Phys. 64541

[16] Stepanow S 1992 J. Phys. A: Math. Gen. 256187

[17] Haronska P and Vilgis T A 1994 J. Chem. Phys. 1013104

[18] For a review, see Isichenko M B 1992 Rev. Mod. Phys. 64 961; Bouchaud J P and Georges A 1990 Phys. Rep. 195127

[19] Fyodorov Y V and Sommers H-J 1997 J. Math. Phys. 381918

[20] Sompolinsky H, Crisanti A and Sommers H-J 1988 Phys. Rev. Lett. 61259

[21] Hatano N and Nelson D R 1996 Phys. Rev. Lett. 77 570; 1997 Phys. Rev. B 568651

[22] Izyumov A V and Simons B D 1999 Europhys. Lett. 45290

[23] Feinberg J and Zee A 1997 Nucl. Phys. B 501 643; Nucl. Phys. B 504579

[24] Edwards S F 1965 Proc. Phys. Soc. 85613

[25] Kleinert H 1995 Path Integrals in Quantum Mechanics, Statistics and Polymer Physics (Singapore: World Scientific)

[26] Sommers H-J, Crisanti A, Sompolinsky H and Stein Y 1988 Phys. Rev. Lett. 601895

[27] Efetov K B 1997 Phys. Rev. Lett. 79491

[28] Chalker J T and Wang J 1997 Phys. Rev. Lett. 791797

[29] See, e.g., Chaikin P M and Lubensky T C 1995 Principles of Condensed Matter Physics (Cambridge University Press)

[30] Lerner I V, private communication

[31] Lifshitz I M 1964 Usp. Fiz. Nauk 83617 [1965 Sov. Phys. - Usp. 7 549]; see also Balagurov B Ya and Vaks V G 1974 Sov. Phys. - JETP 38 968; Lubensky T C 1984 Phys. Rev. A 30 2657; Renn S R 1986 Nucl. Phys. B 275 273; Nieuwenhuizen T M 1989 Phys. Rev. Lett. 62357 